\title{
Philosophiques
}

\section{L'autonomie relationnelle : un nouveau fondement pour les théories de la justice}

\section{Laurence Ricard}

Volume 40, numéro 1, printemps 2013

URI : https://id.erudit.org/iderudit/1018381ar

DOI : https://doi.org/10.7202/1018381ar

Aller au sommaire du numéro

\section{Éditeur(s)}

Société de philosophie du Québec

ISSN

0316-2923 (imprimé)

1492-1391 (numérique)

Découvrir la revue

Citer cet article

Ricard, L. (2013). L'autonomie relationnelle : un nouveau fondement pour les théories de la justice. Philosophiques, 40(1), 139-169.

https://doi.org/10.7202/1018381ar
Résumé de l'article

La notion d'autonomie personnelle joue un rôle central dans les théories politiques contemporaines et, plus spécifiquement, dans les théories de la justice. Or, dans le paradigme libéral dominant, elle est définie par une compréhension rationaliste de l'agent individuel. La présente étude défend la nécessité de redéfinir ce concept d'autonomie à la lumière des développements philosophiques et psychologiques qui ont complexifié notre compréhension de la subjectivité. L'emploi du concept d'autonomie relationnelle développé par certains auteurs féministes et par certains théoriciens de la reconnaissance semble pour ce faire prometteur. Cet article cherche à montrer qu'une compréhension relationnelle de l'autonomie est nécessaire pour expliquer la motivation à la coopération sociale et pour redéfinir la justice et l'injustice de façon à ce qu'elles correspondent à l'expérience sociale vécue. 


\title{
Varia
}

\section{L'autonomie relationnelle: un nouveau fondement pour les théories de la justice'}

\author{
LAURENCE RICARD \\ Université McGill \\ laurence.ricard@mail.mcgill.ca
}

\begin{abstract}
RÉSUMÉ. - La notion d'autonomie personnelle joue un rôle central dans les théories politiques contemporaines et, plus spécifiquement, dans les théories de la justice. Or, dans le paradigme libéral dominant, elle est définie par une compréhension rationaliste de l'agent individuel. La présente étude défend la nécessité de redéfinir ce concept d'autonomie à la lumière des développements philosophiques et psychologiques qui ont complexifié notre compréhension de la subjectivité. L'emploi du concept d'autonomie relationnelle développé par certains auteurs féministes et par certains théoriciens de la reconnaissance semble pour ce faire prometteur. Cet article cherche à montrer qu'une compréhension relationnelle de l'autonomie est nécessaire pour expliquer la motivation à la coopération sociale et pour redéfinir la justice et l'injustice de façon à ce qu'elles correspondent à l'expérience sociale vécue.
\end{abstract}

\begin{abstract}
The notion of personal autonomy plays a central role in contemporary political theories and especially in theories of justice. Generally speaking, the dominant liberal paradigm defines individual agency in a purely rationalistic manner. Against this tradition, the present study argues for the necessity of rethinking and redefining this rationalistic concept of autonomy in light of psychological and philosophical developments that have complicated our understanding of subjectivity. For this purpose, the concept of relational autonomy, developed by feminist authors and some theorists of recognition, is most promising. This article aims to show that a relational understanding of autonomy is necessary to explain motivations for social cooperation and to understand justice and injustice in a way that corresponds to lived social experience.
\end{abstract}

Le libéralisme autant que le libertarisme sont nés de la conviction acquise depuis le début de la modernité que les individus sont autonomes et qu'ils ont donc la capacité de faire des choix, de s'autodéterminer, de fixer leurs propres objectifs et de réfléchir de façon critique sur les normes sociales qui les encadrent. Avec les progrès de la démocratie, aujourd'hui l'emblème politique des pays occidentaux, la notion d'autonomie individuelle est devenue un des piliers de la modernité. Ce faisant, elle est aussi une des prémisses les plus importantes de la réflexion politique contemporaine. En effet, plusieurs

1. Merci à Christian Nadeau, Peter Dietsch et Michel Seymour pour leurs commentaires sur une version précédente de ce texte. 
considèrent à la suite de Kant que l'autonomie est la description la plus fondamentale et universelle de l'être humain ${ }^{2}$. Elle serait donc aussi la façon la plus neutre de décrire l'agent individuel, de manière à travailler avec un concept d'agent qui laisse place aux différentes conceptions compréhensives de l'individu. Chose certaine, étant donné la prédominance du libéralisme au sein des courants de philosophie politique, l'individu autonome est devenu une donnée centrale des théories de la justice. Malgré tout, avec la deuxième vague du féminisme et la critique communautarienne déjà devenue classique, la conception libérale de la personne a été défiée sur plusieurs fronts. Ces critiques reposent principalement sur la conception étroite de l'individu et son aveuglement devant le fait que l'identité personnelle est en grande partie socialement construite. Or, aujourd'hui, une certaine critique que l'on pourrait regrouper sous l'appellation des théories relationnelles va encore plus loin en affirmant que, plus qu'uniquement l'identité personnelle, c'est la façon même d'agir des individus qui est affectée par les relations sociales.

La conception de l'autonomie individuelle au cœur des théories de la justice est non seulement un outil analytique pour comprendre les principes de justice: elle est le fondement même de la conception de la justice qui l'anime. Puisque les théories de la justice proposent différentes formes d'organisation sociale, de répartition des tâches au sein de la société, de rapports entre les individus, de distribution et redistribution des biens, elles fonctionnent nécessairement sur certains présupposés quant à la nature humaine et à ce qui motive chaque individu dans ses décisions. Par conséquent, la conception de l'autonomie individuelle au cœur de chaque théorie de la justice a un impact majeur, non seulement sur les principes de justice qu'elle défend, mais aussi sur la possibilité réelle de répondre aux besoins physiques, psychologiques et moraux des individus. L'adoption d'une conception de l'autonomie plus proche de la réalité vécue des individus permettrait d'élaborer une théorie de la justice plus apte à inspirer la construction d'institutions sociales fonctionnelles qui répondent aux exigences de justice fondamentale.

Ce texte portera sur la nécessité d'employer une notion relationnelle d'autonomie dans les théories politiques. Nous suivrons pour ce faire deux pistes de réflexion. D'une part, selon plusieurs critiques du libéralisme, la conception atomiste et surtout rationaliste de l'individu au fondement des théories de la justice libérales ne tient pas compte du fait que l'agent individuel est constitué par ses relations sociales. Cette position dépasse la critique communautarienne classique, puisqu'elle n'affirme pas uniquement que l'identité individuelle est constituée par les interactions sociales, mais aussi que l'individu en tant qu'agent moral et politique est façonné par les relations interpersonnelles et sociales qu'il entretient avec son entourage et la société

2. C'est le cas entre autres de John Rawls, le chef de file du libéralisme néokantien, mais aussi de plusieurs auteurs à sa suite, comme John Christman. 
à laquelle il appartient. Cette thèse suppose que l'autonomie dépend d'abord du rapport à soi-même, et que ce rapport est constitué en très grande partie par la reconnaissance ou le regard des autres. D'autre part, le fait de caractériser les agents individuels comme étant autonomes joue un rôle fondamental dans l'élaboration des théories de la justice et dans la défense d'un régime démocratique. L'autonomie individuelle sert alors de contrepoids face au pouvoir de la majorité et à l'autorité des institutions. Par conséquent, certains auteurs ${ }^{3}$ pensent qu'il est nécessaire de s'en tenir à une conception procédurale et individualiste de l'autonomie. Deux questions se posent donc: en premier lieu, pourquoi serait-il nécessaire de modifier notre concept d'autonomie de manière à rendre compte de la dimension sociale et affective de l'agent? Ensuite, est-il pensable d'élaborer un tel concept sans qu'il ne soit un paradoxe? C'est-à-dire que, dans la mesure où l'autonomie signifie la capacité de faire des choix et de se déterminer soi-même, le fait de définir l'autonomie de façon plus substantielle n'est-il pas déjà une restriction de l'autodétermination? Ces deux questions serviront de fil conducteur à notre réflexion.

Notre projet n'est pas d'élaborer une conception viable de l'autonomie relationnelle 4 . En effet - et c'est ce qui rend le travail d'autant plus ardu le concept d'autonomie relationnelle est, dans la littérature actuelle, tout au plus un projet. On y fait souvent allusion dans de multiples débats, entre autres dans les domaines éthiques et juridiques, mais ceux-ci se fondent généralement sur une conception mouvante de la nature relationnelle du sujet ${ }^{5}$. Nous ne comptons pas ici trancher la question en proposant un concept unifié de l'autonomie relationnelle. L'objectif est plutôt de démontrer qu'il est possible d'élaborer un concept d'autonomie personnelle qui tienne compte de l'interdépendance des individus sans toutefois surdéterminer les caractéristiques de l'autonomie. Le pari est alors celui de défendre la possibilité d'un concept formel d'autonomie, donc flexible et non rigide, qui tienne compte de la nature indéniablement interdépendante des êtres humains.

3. Pensons entre autres à John Christman. Cf. J. Christman, «Autonomie individuelle et moi social», in Comment penser l'autonomie: entre compétences et dépendances, sous la dir. de M. Jouan et S. Laugier, Paris, Presses universitaires de France, 2009.

4. Axel Honneth propose un "recognitional account of autonomy" qu'il associe luimême explicitement aux travaux féministes sur l'autonomie relationnelle. (Cf. "Autonomy, Vulnerability, Recognition, and Justice», J. Anderson et A. Honneth, in Autonomy and the Challenges to Liberalism: New Essays, sous la dir. de J. Christman et J. Anderson, Cambridge, UK; New York, Cambridge University Press, 2005.)

5. Les divers emplois et diverses conceptions de l'autonomie relationnelle dans le contexte des débats éthiques sont bien représentés dans Relational autonomy: Feminist perspectives on autonomy, agency, and the social self, C. Mackenzie et N. Stoljar (dir.), Oxford University Press, USA, 2000. On retrouve aussi la conception relationnelle du sujet dans le monde académique juridique: Contextual Subjects: Family, State and Relational Theory. R. Leckey, University of Toronto Press, 2008; "Relational Contract Theory: Challenges and Queries » I. R. Macneil, Nw. UL Rev. 94 (I999) 877. 
L'analyse que nous mènerons portera sur un concept essentiellement descriptif de l'autonomie. Afin de cerner cet objet, certaines distinctions entre diverses approches de l'autonomie sont de mises. Dans l'introduction à leur ouvrage sur l'autonomie, Christman et Anderson mettent en contraste l'autonomie de base de l'autonomie idéale ${ }^{6}$. La première représente un certain niveau de gouvernement de soi-même nécessaire pour obtenir le statut d'agent moral ou de sujet politique. La deuxième va plus loin, en jouant le rôle d'une idée régulatrice sans toutefois être nécessaire (du moins, pour certains auteurs) à l'obtention des droits, de la responsabilité individuelle et autres statuts conférés par la reconnaissance de l'autonomie de base. L'autonomie relationnelle se situe à la frontière de ces deux types d'autonomie. Nous verrons que, bien qu'elle se classe dans la seconde catégorie, elle a une influence profonde sur la façon dont on conçoit la première, puisqu'elle dépasse cette distinction. L'autonomie relationnelle remet en cause non pas la façon dont on caractérise les individus comme autonomes afin de leur conférer des droits, mais bien plutôt la façon dont on conçoit l'individu comme agent. Elle est en ce sens un outil pour la théorie de l'action. Les auteurs qui s'intéressent à ce concept cherchent à mieux comprendre la psychologie morale et sociale des individus afin de créer des modèles de justice qui répondent de façon plus adéquate au besoin d'émancipation individuelle. Ce besoin est, selon eux, intimement lié à la façon dont l'individu vit ses relations interpersonnelles et sociales. Il s'agit donc de voir en quoi un concept descriptif et donc formel de l'autonomie a nécessairement une incidence sur le projet normatif pour lequel il sert d'outil théorique.

Dans un premier temps, nous montrerons la nécessité d'adopter un concept d'autonomie relationnelle. Nous commencerons par évaluer l'argument du moi dialogique, tel qu'exposé par Charles Taylor dans son ouvrage Les sources du moi. Nous nous tournerons ensuite vers des études plus empiriques sur les relations d'interdépendance entre les individus, en nous intéressant plus spécifiquement à une théorie maintenant largement adoptée à la fois par les chercheurs et les cliniciens en psychologie, soit la théorie de l'attachement. Nous nous intéresserons aussi aux théories neuropsychologiques selon lesquelles le rapport entre émotion et raison est plus intime que ce qui est généralement présupposé. Enfin, nous pourrons nous tourner vers les arguments philosophiques d'Axel Honneth en faveur d'une autonomie «décentrée », qui relève de sa théorie de la reconnaissance comme cadre explicatif des rapports sociaux fondamentaux. Dans un deuxième temps, nous démontrerons qu'il est possible d'adopter un concept d'autonomie relationnelle qui ne limite pas la capacité d'autodétermination des individus et qui respecte les fondements politiques démocratiques. Nous verrons donc d'abord la place conceptuelle que devrait prendre une telle notion dans l'élaboration

6. Autonomy and the Challenges to Liberalism: New Essays, J. Christman et J. Anderson (dir.), Cambridge, UK, Cambridge University Press, 2005, p. 2. 
des théories de la justice, pour nous pencher ensuite sur le problème de la surdétermination de la notion d'autonomie.

\section{Vers une définition formelle de l'autonomie relationnelle: les raisons philosophiques et empiriques de l'adoption de ce concept}

La critique la plus répandue du sujet libéral, qui a pris forme durant les dernières décennies, porte sur l'oubli de l'interdépendance des personnes et de la nature sociale de l'identité individuelle dans le libéralisme contemporain. Deux volets de cette critique peuvent cependant être distingués. Le premier volet est le moins controversé. Il s'agit de l'affirmation d'un «moi social» en opposition à un "moi désincarné ». Selon cette portion de la critique, l'individu n'est pas uniquement un agent rationnel indépendant. Son identité personnelle se construit en fonction des relations sociales qu'il entretient. Les auteurs libéraux ont, pour la plupart, accepté cette critique. Rawls lui a d'ailleurs répondu par la redéfinition de sa théorie dans Libéralisme politique. Le deuxième volet de la critique a fait naître des réactions beaucoup plus mitigées, puisqu'il cherche à remettre en cause notre compréhension non seulement du contenu de l'identité personnelle, mais de la structure même de l'agent individuel. C'est à l'analyse de cette deuxième partie de la critique que je me consacrerai. Elle suppose que l'interdépendance sociale joue un rôle non seulement dans la construction de l'identité personnelle, mais aussi dans les possibilités d'action et d'autonomie de l'individu. Alors que la critique communautarienne classique montre comment les relations interpersonnelles sont constitutives du moi (de l'identité individuelle), nous nous demanderons plutôt comment elles sont constitutives du je (de la psychologie de l'agent démocratique). Pour donner des assises à la théorie relationnelle de l'autonomie, on doit d'abord démontrer que l'individu est constitué par ses relations interpersonnelles, mais aussi que ces relations ont une influence sur ses actions. Toutefois, si l'on veut préserver une conception de l'individu comme être capable de rationalité (ce que la préservation de la notion d'autonomie commande), il nous faut montrer en quoi la rationalité est elle-même profondément influencée par l'interaction des individus avec leurs semblables, en raison de leurs réactions émotives face aux expériences vécues.

\subsection{La construction dialogique du moi}

Charles Taylor est un des penseurs qui a le plus contribué à l'évaluation des conséquences de l'attachement moderne (et typiquement libéral) à la notion d'autonomie. Une de ces conséquences premières est, selon lui, la minceur de la notion d'identité personnelle dans la pensée politique dominante. En réponse à ce problème, sa présentation de la construction dialogique du moi nous permet de comprendre que les questions du moi (l'identité) et du je (l'agent) peuvent être interprétées comme étant deux versants d'une même problématique. Par conséquent, accepter la construction sociale de l'identité personnelle revient à admettre la nécessité de repenser l'autonomie individuelle. 
Lorsque Taylor parle du moi, il désigne l'identité personnelle, cette définition de soi-même essentielle à toute quête d'autonomie. Or c'est à notre identité, à ce moi, que nous nous rapportons lorsque nous prenons une décision autonome. L'affirmation du caractère fondamentalement dialogique de ce mo $i$ a donc une incidence certaine sur la façon dont on peut penser l'autonomie. Si, en effet, on adhère à la description que Taylor fait de l'identité personnelle (du moi), il est nécessaire d'accepter la notion relationnelle de l'autonomie, au moins en tant que concept descriptif.

Ce qu'affirme Taylor, c'est que le moi ne peut être pensé en dehors des "réseaux d'interlocution" qui le constituent ${ }^{7}$. En fait, un moi est toujours un moi qui se pense, puisque l'identité personnelle est au moins en partie constituée de l'interprétation que l'on se fait de soi-même. La façon dont je me perçois, dont je me décris, constitue en partie ce que je suis. C'est d'ailleurs ce qui en fait un objet d'étude dans une classe à part, selon Taylor, dans la mesure où il n'est pas indépendant des interprétations que l'on peut en faire. Cette affirmation de la constitution de l'identité par le récit qui en est fait est essentielle à la notion d'autonomie relationnelle, puisqu'il s'agit d'une première étape pour comprendre où s'effectue le passage entre les rapports sociaux et la façon dont l'agent se conçoit comme agent. Effectivement, la façon dont je décris et interprète mon identité est partie intégrante de mon moi. Mais en même temps, je ne peux effectuer cette description qu'avec les outils linguistiques de la communauté à laquelle j'appartiens à un moment donné: "étudier les personnes, c'est étudier des êtres qui existent uniquement dans un certain langage ou qui en sont partiellement constitués ${ }^{8}{ }^{\prime}$. En affirmant la primauté du langage sur la constitution du moi, Taylor explicite la thèse de la constitution de l'identité individuelle par la narration. Bien qu'il donne aux institutions langagières un rôle primordial dans la construction de l'identité personnelle, Taylor rejette toutefois la thèse poststructuraliste de la fin de la subjectivité. La raison en est qu'il cherche à préserver la notion d'autonomie. Son argument est plutôt que la constitution du moi résultant d'une médiation sociale du langage n'est pas incompatible avec l'idée moderne d'autonomie personnelle. Il souligne d'ailleurs que «l'autonomie moderne ne constitue pas une négation du fait qu'un moi existe seulement parmi d'autres "moi" ". Reconnaissant la possibilité d'une originalité personnelle, Taylor nous dit:

Un être humain peut toujours être original, prendre ses distances par rapport à la pensée et à la vision du monde de ses contemporains, voire ne pas être compris. Mais cette compulsion à développer une vue personnelle des choses se trouvera inhibée et se perdra ultimement dans la confusion intérieure, à moins

7. Les sources du moi: la formation de l'identité moderne, C. Taylor, traduction de

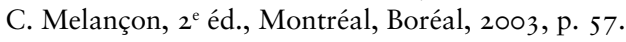

8. Ibid., p. 55.

9. Ibid., p. 56. 
qu'elle ne se situe d'une façon ou d'une autre en relation avec le langage et la vision des autres ${ }^{10}$.

Cette citation nous ramène à la distinction entre le moi, soit l'identité personnelle, et le je, l'agent humain ${ }^{11}$. On voit bien ici, à travers cette formulation de l'autonomie, qu'il est impossible de dissocier les deux. Si Taylor parle toujours explicitement du moi, ce passage réfère bel et bien au je, c'està-dire à ce qui, en moi, est capable d'agir, d'élaborer et d'énoncer des idées, de créer, bref, d'être agent. Ce que Taylor semble nous dire ici, c'est que la possibilité même de devenir un agent autonome dépend de l'usage du langage socialement partagé et des visions du monde que le langage implique. Pour être capable soi-même d'abord de former puis d'énoncer ses préférences, il faut avoir les outils linguistiques pour le faire. Ce qui est sous-entendu dans ce passage, c'est que la rationalité, qui fait de nous des êtres potentiellement autonomes, dépend pour être mise en œuvre du langage. Tant qu'une certaine forme de langage n'a pas mis en forme les impressions, émotions, sentiments ou intuitions originales qui se développent dans l'individu particulier, on ne peut pas parler d'autonomie au sens de développement d'un esprit critique.

Si l'autonomie est effectivement liée à la réflexion critique ${ }^{12}$, deux versions d'un même argument peuvent être avancées. La plus forte consiste à montrer le lien entre la réflexion critique et le dialogue au sens propre. Je crois que cette version est défendable, entre autres en prenant pour exemple l'histoire de la philosophie. On n'innove qu'en référence à ce qui existe déjà. La réflexion est un reflet modifié grâce à un nouvel angle d'observation, mais elle demeure un reflet de ce qui a déjà été dit. Cet argument se suffit à

10. Ibid., p. 58-59.

11. Taylor reprend ici la distinction d'abord faite par G. H. Mead. Toutefois, et cette différence est cruciale pour notre compréhension de l'autonomie, il refuse de considérer, à la suite de Mead, que le «je» est une sorte de coquille vide indépendante du «moi», un simple principe d'originalité: "What [Mead's conception of the "I"] fails to capture is the way in which the "I" is constituted as an articulate identity defined by its position in the space of dialogical action. [...] The conversation between the "I" and the "me," or between one's own selfgenerated transformations of the offered scenarios and their original form, is not between and introjected identity and some unformed principle of spontaneity. It is more a matter of gradually finding one's own voice as an interlocutor, realizing a possibility which was inscribed in the original situation of dependence in virtue of its dialogical form. " C. Taylor, "The Dialogical Self» dans R. E. Goodman et W. R. Fischer (dir), Rethinking Knowledge: Reflections Across the Disciplines Albany, State University of New York, I995, de 57-66 à 64-65.

12. Certains diront que penser l'autonomie en termes de réflexion critique est déjà une surdétermination du concept, mais cet argument est faible. On ne peut travailler avec un concept vide de signification. Si l' «autonomie » telle que définie dans le cadre de notre problématique veut dire la possibilité de faire des choix par rapport à sa propre vie, la notion de choix qui fait partie de cette définition implique une forme de rationalité. Or on voit mal quel type de rationalité autre pourrait définir le choix autonome, si ce n'est la capacité à exercer une réflexion critique sur les conditions du choix. 
lui-même et prouve la nécessité de repenser le concept d'autonomie en tant qu'obsession d'une authenticité détachée de toutes dépendances. Toutefois, il mériterait plus d'attention qu'on peut ici lui en consacrer. On peut donc aussi se contenter de la version plus faible, peut-être plus facile à accepter, qui consiste à montrer que les outils linguistiques sont indispensables à toute réflexion critique, ou même à n'importe quelle sorte de rationalité. Je me contenterai de cette version plus faible, pour montrer dans la prochaine section que l'attachement émotionnel et les relations sociales qui le sous-tendent sont parties prenantes du développement de la rationalité humaine et, plus spécifiquement, des capacités linguistiques et de l'intelligence sociale.

\subsection{L'apport de la psychologie: la neuroscience sur le rapport entre émotions et raison, et la théorie de l'attachement}

Le rapport intime d'influence mutuelle entre émotions et cognition n'est plus à démontrer dans les multiples sphères de la recherche psychologique. Malgré la diversité des approches dans ce domaine, de plus en plus de courants se rallient à l'idée que non seulement émotion et raison ne sont pas deux termes opposés, mais bien qu'ils sont en interaction constante. Je ne compte pas faire ici un rapport exhaustif des travaux en psychologie sur le rôle des émotions, des sentiments et des liens affectifs dans l'évolution cognitive des individus, mais je souhaite plutôt montrer que cette tendance représente un courant dominant et qu'elle pointe vers la validation de l'hypothèse selon laquelle le processus de cognition, et donc, la prise de décision nécessaire à toute notion d'autonomie, est intimement lié au bagage émotionnel et social d'un individu. Cette conception de l'individu se voit autant dans le domaine de la neuropsychologie que dans la théorie de l'attachement.

\subsubsection{Neurosciences, émotions et cognition}

En neurobiologie, de plus en plus d'études sur les émotions et leur rôle dans la cognition sont menées depuis une décennie, et elles démontrent l'imbrication des deux concepts:

Depuis ces dix dernières années, grâce à l'appui des données expérimentales et des progrès opérés en neuro-imagerie, les études des émotions en neurosciences sont en pleine expansion. Jetant le trouble sur une longue période de rationalisme, certains de ces travaux nous ont même fait comprendre que l'émotion et la raison ne sont certainement pas des notions aussi opposées que ce que l'on pensait mais que, au contraire, l'émotion constitue une part essentielle des processus de raisonnement et de prise de décision ${ }^{13}$.

On démontre entre autres dans ces études que le cortex orbito-frontal, une partie à l'avant du cerveau, est en premier lieu un centre de traitement

13. «Neurosciences et affects», B. Giffard et B. Lechevalier, Champ psychosomatique 4I, $\mathrm{n}^{\circ}$ I (2006), p. II. 
émotionnel, mais joue aussi un rôle important «dans la prise de décision, et dans la mémorisation des souvenirs liés aux récompenses et aux punitions ${ }^{14}$ ». Cette zone du cerveau est associée à la fois aux émotions suscitées par les interactions sociales et aux processus de raisonnement qui mènent à la prise de décision ${ }^{15}$. L'observation de certains cas cliniques a permis de confirmer cette hypothèse. Le plus célèbre est celui de Phineas Gage, qui a survécu à un accident qui lui a transpercé le cortex préfrontal et n'a subi aucun dommage perceptible, sauf dans la faculté de prendre des décisions en concordance avec les valeurs morales qu'il avait internalisées et les dictats de l'environnement social. Selon Giffard et Lechevalier, qui ont élaboré une revue de l'état des connaissances en neurobiologie relativement aux liens entre émotions et raison, ce type de cas illustre bien que «les facultés de raisonner et d'éprouver des émotions vont de pair, et leur affaiblissement tranche nettement avec un profil neuropsychologique qui montre une parfaite préservation de la mémoire, de l'attention, du langage et de l'intelligence ${ }^{16}{ }^{\prime}$.

Selon des recherches menées sur cette partie spécifique du cerveau, les individus associeraient des réactions physiques émotionnelles à certaines situations vécues qui leur permettent d'enclencher un processus de prise de décision qui tienne compte de leurs expériences passées.

Ces marqueurs somatiques sont élaborés au cours des processus d'éducation et de socialisation. En effet, tout au long de la vie, depuis l'enfance, nous associons à nos actes, à nos choix, des représentations somatiques qui sont en fait des manifestations émotionnelles vécues comme conséquences de nos actes ${ }^{17}$.

Les «marqueurs somatiques ${ }^{18}$ » agissent comme guides qui permettent d'éliminer spontanément les options menant à de mauvaises conséquences. C'est-à-dire que nos relations sociales créent des émotions qui nous permettent par la suite de prendre des décisions, lesquelles seront qualifiées de rationnelles dans la mesure où l'on considérera qu'elles reposent sur une évaluation cognitive des coûts et bénéfices à partir des informations disponibles. La propriété de ces marqueurs émotionnels qui nous intéresse ici est surtout leur caractère éminemment social. L'émotion qu'ils suscitent est une médiation entre l'expérience sociale vécue et le processus rationnel de décision. Giffard et Lechevalier concluent d'ailleurs leur état des lieux de la neuroscience sur ce sujet par le constat suivant: «L'émotion, loin d'être le fardeau

14. Ibid., p. 2 I.

15. Plusieurs études tendent à démontrer que c'est le cortex orbito-frontal qui permet la compréhension d'autres personnes à travers les émotions et sentiments qui accompagnent l'interaction sociale. Cf. "Cognitive Neuroscience of Human Social Behaviour », R. Adolphs, Nature Review Neuroscience 4 (2003), p. I72.

16. "Neurosciences et affects", Giffard et Lechevalier, p. 23.

17. Ibid., p. 24.

18. Le terme a été consacré par A. R. Damasio dans L'erreur de Descartes, A. R. Damasio, Paris, Éditions Odile Jacob, I994. 
de la raison, devient l'auxiliaire de la raison. On comprend ainsi que la prise de décision ne peut se baser uniquement sur la raison pure [...]. ${ }^{19}$ " La prise de décision dépend de l'intériorisation de codes sociaux par le biais d'émotions guidant le choix entre les différentes options qui définissent le contexte de décision ${ }^{20}$. Et, comme nous allons le voir sous peu, cette connexion entre rapports sociaux, émotions et développement cognitif est si profonde qu'elle commence à se faire en très bas âge.

\subsubsection{La théorie de l'attachement}

La notion d'autonomie relationnelle a pris racine entre autres dans le féminisme. Les théories du care, qui s'inscrivent dans la pensée féministe, se fondent sur le rapport de dépendance le plus profond que tous les humains peuvent expérimenter, soit le rapport entre la mère (ou une figure substitut permanente) et son enfant. Ce rapport a été analysé et expliqué en psychologie par la théorie de l'attachement. John Bowlby et Mary Ainsworth, les initiateurs de cette théorie, sont de nos jours des pionniers de la recherche clinique en psychologique telle qu'elle se pratique aujourd'hui, leur œuvre étant un des piliers de la psychologie contemporaine. Leurs travaux ont démontré que, plus qu'un simple résultat de la dépendance physique du nourrisson par rapport à la figure maternelle, l'attachement est un besoin primaire au même titre que les besoins physiques ${ }^{21}$.

La théorie de l'attachement a donné et donne encore lieu à de nombreuses découvertes dans notre compréhension du développement de l'enfant, mais sa portée dépasse le monde de l'enfance. Cette théorie a confirmé que les concepts de soi (self) et de l'autre se forment de façon interdépendante, c'est-à-dire que selon les expériences au sein de la relation d'attachement, l'enfant va se forger une certaine conception de la figure d'attachement (présente, aimante, ou à l'inverse fuyante, détachée), mais va aussi à la fois construire sa propre conception de lui-même comme étant digne d'amour et d'attention ou non ${ }^{22}$. Ce postulat, nous y reviendrons, joue en faveur de la théorie de la reconnaissance développée par Axel Honneth, qui suppose un lien entre notre rapport à nous-mêmes et les réactions de notre environnement social. Des liens ont aussi été tissés entre les modèles d'attachement et les capacités cognitives des enfants, ce qui laisse entrevoir, comme le suggé-

19. «Neurosciences et affects », Giffard et Lechevalier, p. 52.

20. Pour Jerome Bruner, psychologue cognitiviste, la mise en contexte des émotions, de la cognition et de l'action dans le système culturel qui lui donne sens est essentielle à la compréhension des trois concepts, puisque c'est le contexte social qui les intègre et leur donne sens. Cf. Actual Minds, Possible Worlds, J. S. Bruner, Cambridge, Mass., Harvard University Press, I986.

21. Voir, entre autres, L'attachement, J. Bowlby, Paris, Presses Universitaires de France, I969.

22. «Attachment Theory: Retrospect and Prospect ", I. Bretherton, Monographs of the Society for Research in Child Development 5O, no. I (I985), p. I2-I3. 
raient les recherches en neurosciences résumées plus haut, que les affects en jeu dans la relation d'attachement jouent un rôle dans la capacité de cognition des individus. Certaines recherches montrent en particulier que les enfants qui ont des relations sociales fortes sont plus stimulés sur le plan cognitif et développent, entre autres, de meilleures capacités linguistiques ${ }^{23}$. On retrouve donc dans la théorie de l'attachement le lien entre le développement affectif d'un individu et son développement cognitif ${ }^{24}$.

La neuroscience a elle aussi confirmé l'importance de l'attachement pour le développement des capacités cognitives. Les travaux de Allan Schore, entre autres, démontrent l'effet des émotions suscitées par la relation d'attachement sur la formation de l'hémisphère droit du cerveau. En conclusion d'un article sur le sujet, Schore affirme: «L'information qu'offrent ces travaux suggère que l'intelligence émotionnelle ou sociale dépend fortement du fonctionnement de l'hémisphère droit du cerveau, et que cette capacité est le résultat d'un attachement sécurisant (secure attachment) et la composante d'une santé mentale adaptative chez les jeunes enfants, les enfants, les adolescents et les adultes ${ }^{25}$.»Par ailleurs, dans les recherches en neuropsychologie, il a été avancé que l'on peut distinguer l'intelligence sociale de l'intelligence générale, l'intelligence sociale faisant référence à l'habileté de se représenter les états mentaux des autres, autant dans des situations sociales complexes que dans des relations intimes ${ }^{26}$. Lorsque nous faisons référence en philosophie à la rationalité déployée pour gérer les relations sociales, nous faisons référence à ce type d'intelligence sociale. À la lumière de ces recherches, il devient évident qu'on ne peut plus penser la façon dont l'individu fait des choix, donc est autonome, indépendamment des relations sociales qui le constituent.

23. "Attachment, Intelligence, and Language: A Meta-analysis», M. H. van IJzendoorn, J. Dijkstra et A. G. Bus, Social Development 4, $\mathrm{n}^{\circ} 2$ (I995). Il faut bien voir toutefois que la conclusion des auteurs est que l'attachement n'est pas un facteur affectant l'intelligence des individus, mais bien plutôt un gage d'amélioration du développement des capacités cognitives.

24. Sur les travaux récents dans le cadre de la théorie de l'attachement, voir entre autres: The Philosophical Baby: What Children's Minds Tell Us About Truth, Love, and the Meaning of Life, A. Gopnik, Ist, New York, Farrar, Straus and Giroux, 2009. Voir aussi les travaux de Allan N. Schore (référence plus bas) et Daniel Stern, entre autres, The Interpersonal World of the Infant: A View from Psychoanalysis and Developmental Psychology, D. N. Stern, New York, Basic Books, I985.

25. A. N. Schore, "Effects of a Secure Attachment Relationship on Right Brain Development, Affect, Regulation, and Infant Mental Health ", Infant Mental Health Journal 22, $\mathrm{n}^{\circ} \mathrm{I}-2$ (200I), p. 48. "The data offered in this work suggest that emotional or social intelligence relies heavily upon right brain function, and that this capacity is an outcome of a secure attachment and a central component of adaptive infant, child, adolescent, and adult mental health."

26. Ibid. 


\subsubsection{La raison différenciée}

Les liens sociaux, que ce soit les liens très intimes de la figure d'attachement avec un enfant ou le rapport de la société à l'individu, ont un impact direct sur la façon dont l'individu met en œuvre sa rationalité. Dans un ouvrage portant sur les effets des relations de compétition et des situations de risques sur la réciprocité et la coopération sociale, Peter Marris fait appel à la description que Piaget fait de la pensée:

Piaget décrit la structure de la pensée comme une forme distinctivement humaine de l'organisation autorégulatrice. La pensée, comme les autres capacités des êtres humains, émerge d'un héritage génétique, se développe en interaction avec son environnement, évolue et se transforme, mais toujours dans le but de maintenir l'intégrité de sa structure - assimiler de nouvelles informations, s'adapter aux incohérences et se réconcilier avec les anomalies ${ }^{27}$.

À chaque fois qu'une décision est prise, les éléments décisionnels qui entrent dans le calcul rationnel sont construits par la mémoire de l'individu, par les représentations qu'il s'est créées dans le passé et, nécessairement, par les émotions qu'ont suscitées les expériences passées. Lors du processus de décision, il y a deux éléments principaux qui sont pris en considération : la signification sociale des données en jeu dans la décision, et la signification émotive, donc très personnelle et individuelle, de ces mêmes données.

C'est ce qu'explique la psychiatre Françoise Schenk, dans sa caractérisation de la décision:

La décision suppose un choix explicite entre différentes options. La décision rationnelle implique une optimalité de ce choix, basé sur l'intégration des données pertinentes. Comme le souligne l'économiste Herbert Simon, cette optimalisation est manifestement limitée par les informations disponibles. Elle est également limitée par le fait que ces données ont inévitablement les deux faces invoquées plus haut, à savoir leur référent dans l'environnement du sujet et leur nature intime, leur signification pour le sujet. Pour le sujet, leur statut est celui d'une représentation, pas d'une grandeur mesurée "objectivement». Cette représentation repose sur une extraction de contrastes, sur une échelle relative et non absolue, ce qui n'en réduit pas la précision mais en module la saillance, la valeur peut-être ${ }^{28}$.

27. The Politics of Uncertainty: Attachment in Private and Public Life, P. Marris, New York, Routledge, I996, p. I9. "Piaget describes the structure of thought as the distinctively human form of this self-regulative organization. Thought, like other capacities of human beings, grows out of a genetic endowment, develops in interaction with its environments, evolves and transforms itself, yet always with the aim of maintaining the integrity of its structure-assimilating new information, accommodating to inconsistencies, reconciling anomalies.»

28. "Les émotions de la raison", F. Schenk, Revue européenne des sciences sociales, tome XLVII, ${ }^{\circ}$ I 44 (2009), p. I 58. 
Les représentations à partir desquelles s'effectue une décision rationnelle ne sont donc jamais, à proprement dit, objectives. Elles sont plutôt le produit des diverses expériences de l'individu et du souvenir qu'il en $a^{29}$. Or la mémoire est formée et déformée selon les affects associés à certaines situations. La décision rationnelle n'est donc jamais ultimement rationnelle qu'au regard des éléments non rationnels qui l'informent. Ces éléments reposent, dans les relations sociales, sur la caractérisation de la situation relationnelle.

La difficulté d'extraire la rationalité du processus de décision est encore plus grande lorsqu'il s'agit d'interactions sociales. Il est essentiel de voir à quel point la rationalité est tributaire de nombreuses influences et peut difficilement, dans le cas de la gestion de relations sociales (ce qu'est nécessairement la philosophie politique), être un fondement d'universalité. Si la rationalité théorique, celle de l'abstraction et des sciences dures comme les mathématiques, peut donner lieu à des conceptualisations indiscutables et nous pouvons même douter que de telles disciplines soient entièrement soustraites au débat - , il n'en est pas de même dans la façon dont nous usons de notre rationalité pour participer à la réalité sociale. Chacune de mes décisions est le produit de l'information dont je dispose. Or, dans une situation de relation interpersonnelle plus encore que dans n'importe quelle autre situation, l'autre, élément essentiel dans ma délibération, est inconnaissable. Je ne peux que l'appréhender par la projection de ce que je suis, un semblable, et par mon expérience de mes relations antérieures.

On ne peut que constater que la façon dont nous usons de rationalité dans les situations d'interaction sociale dépend de la manière dont on conçoit les signaux que nous envoient nos semblables et, par conséquent, dépend en grande partie du regard de l'autre. Tous les éléments d'une relation interpersonnelle ou sociale participent à la création de l'individu en tant qu'agent, puisqu'ils suscitent autant d'émotions, d'expériences, de souvenirs, de sentiments qui informent les décisions et les actes individuels. C'est ce rapport entre la formation de mon identité en tant qu'être autonome et de la reconnaissance dont l'autre me rend digne que la théorie de la reconnaissance conceptualise.

\subsection{La théorie de la reconnaissance}

Axel Honneth a puisé dans l'héritage hégélien et dans la psychologie sociale (s'attardant entre autres à la théorie de l'attachement) afin d'orienter les conclusions que nous venons de voir vers la possibilité de modifier la façon dont on approche la théorie politique contemporaine. L'idée principale au cœur de la théorie de Honneth est que les expériences de reconnaissance et la relation d'une personne avec elle-même sont connectées dans un schéma

29. C'est aussi ce que soutient R. J. Dolan dans ses recherches en neurosciences. Cf. «Emotion, Cognition and Behaviour», R. J. Dolan, Science 298, nº 5596 (2002). 
intersubjectif de construction de l'identité personnelle. Cette affirmation fondamentale permet à Honneth de concevoir les conflits sociaux comme étant toujours déjà moraux, montrant qu'ils résultent toujours d'une forme de déni de reconnaissance qui a un impact direct sur la façon dont l'individu se conçoit lui-même (et, ultimement, conçoit la société) ${ }^{30}$. Honneth et Anderson soulignent, dans un article portant sur l'autonomie, que l'insistance libérale à considérer l'autonomie comme une forme d'indépendance a mené à une construction erronée des demandes de justice sociale ${ }^{31}$. La conception de l'autonomie sur laquelle les deux auteurs s'appuient dans ce texte est décrite comme "une capacité qui n'existe que dans le contexte de relations sociales qui la supportent et seulement en conjonction avec le sentiment intérieur d'être autonome ${ }^{32}$ ». La prémisse sur laquelle ils s'appuient pour expliquer une telle position est que l'autonomie ou la compétence des agents nécessite certaines formes de relation à soi-même (comme on l'a vu un peu plus haut lors de la description de la conception morale de l'autonomie), en particulier la confiance en soi, le respect de soi et l'estime de soi. Or ces trois types de rapport à soi-même sont conditionnels à l'attitude d'autres personnes qui les entretiennent par leur reconnaissance de l'individualité, la particularité et l'importance de l'individu.

Cette prémisse est confirmée par les recherches psychologiques que nous avons décrites plus haut, peut-être plus spécifiquement par la théorie de l'attachement. Dans la reconstruction des capacités nécessaires à l'autonomie, chacun de ces rapports à soi assure certaines compétences individuelles. La confiance en soi permet à l'individu de développer des capacités de perception justes de ses expériences, qui lui permettent de délibérer par la suite. Par exemple, le fait d'avoir subi des traumatismes dus à des sévices physiques peut amener une personne à ne plus faire confiance aux signaux que lui envoie son propre corps. Le respect de soi a plutôt trait à la capacité de traiter l'information nécessaire à la délibération et, éventuellement, à l'action libre. En ce sens, les droits juridiques garantissent ce type de relation à soi-même, puisqu'ils délimitent un champ d'action individuel qui ne peut être empiété par d'autres. Quant à l'estime de soi, elle relève plutôt de la nécessité de donner sens à nos actions:

Il y a au moins une tension entre le fait d'adopter un style de vie et le fait de se penser comme faisant quelque chose qui a du sens. Et, [...] être capable de trouver un sens à ce que l'on fait est intimement lié effectivement au fait d'agir. [...] À cause de la façon dont ils peuvent diminuer l'estime de soi, les modèles systématiques de dénigrement posent par conséquent problème, non seulement

30. The Struggle for Recognition: The Moral Grammar of Social Conflicts, A. Honneth, traduction de J. Anderson, Cambridge, Mass., Polity Press, I995, p. I2 I.

31. Anderson et Honneth, "Autonomy, Vulnerability, Recognition, and Justice», p. I29.

32. Ibid. "Autonomy is a capacity that exists only in the context of social relations that support it and only in conjunction with the internal sense of being autonomous. » 
pour le bonheur ou l'identité, mais aussi pour l'agentivité même de ceux qui en sont affectés ${ }^{33}$.

Par conséquent, on peut voir que pour être un agent autonome au sein de la société, certaines capacités sont requises. Elles relèvent en partie du rapport à soi, mais ce rapport est constamment façonné par l'environnement social dans lequel l'individu évolue. On ne peut donc pas considérer l'autonomie comme une caractéristique simple et première de l'individu. Celleci ne peut exister indépendamment de la reconnaissance interpersonnelle et sociale qui, d'une part, nous confirme comme êtres autonomes et, d'autre part, confère un sens aux actions que nous posons. On peut aussi conclure par extension que l'adoption d'un schème normatif de reconnaissance par la société assurerait une démocratie qui ne soit pas factice. En effet, pour que les citoyens participent à la mesure de leur potentiel à la société, on doit garantir les meilleures conditions possibles pour le développement et l'actualisation de leur autonomie.

Cette façon de concevoir l'autonomie motive Anderson et Honneth à élaborer une critique des tenants d'un libéralisme procédural et, entre autres, de Rawls et de son voile d'ignorance. Un des points intéressants de leur critique est leur affirmation selon laquelle il semble arbitraire de choisir la rationalité instrumentale comme caractéristique de l'individualité lors de la description de la position originelle. Ils suggèrent plutôt que les individus en position originelle devraient partager une compréhension des besoins de reconnaissance individuelle pour élaborer une théorie de la justice digne de ce nom. Ils vont même plus loin en affirmant: "peut-être que le problème le plus fondamental en jeu ici est relatif au degré auquel on devrait faire appel à des conceptions quasi-empiriques de l'être humain dans le développement d'une conception de la justice pour les sociétés libérales ${ }^{34}{ }$. C'est précisément ce que nous tentons de démontrer ici.

\section{Pourquoi l'autonomie relationnelle en philosophie politique?}

Même si de nombreuses études en psychologie et de nombreux arguments philosophiques peuvent nous convaincre de la nature relationnelle de l'autonomie, une question centrale demeure: pourquoi serait-il pertinent d'utiliser ce concept dans le contexte de l'élaboration des théories politiques et, plus précisément, des théories de la justice? En effet, si la notion d'autonomie

33. Ibid., p. I37. "There is at least a tension between pursuing that way of life and thinking of oneself as doing something that makes sense. And, [...] being able to make sense of what it is we are doing is intimately tied up with actually doing it. [...] Because of the way they can undermine self-esteem, systematic patterns of denigration thus pose a threat not merely to the happiness or identify but to the agency of those affected."

34. Ibid., p. I4I. «But perhaps the more fundamental issue at stake here has to do with the degree to which we should appeal to quasi-empirical aspects of human personhood in developing a conception of justice for liberal societies. » 
relationnelle commence à s'établir dans des champs comme la philosophie morale ou l'éthique, elle n'a pas beaucoup été discutée en rapport avec la philosophie politique. L'une des raisons principales, probablement, en est que le concept d'autonomie individuelle joue un rôle tout particulier dans les théories politiques. Le domaine politique est celui du fragile équilibre entre collectivité et individualité. Repenser la façon dont on conçoit cette sphère individuelle est donc un exercice très sérieux qui doit être abordé avec minutie si on ne veut pas ouvrir la porte à des dérives politiques qui mettraient en péril certains acquis que l'individualisme moderne a pu implanter dans nos sociétés. C'est pourquoi, dans cette deuxième partie, nous montrerons la faisabilité de développer une conception formelle de l'autonomie relationnelle et l'utilité qu'un tel concept peut avoir pour les théories politiques contemporaines. Nous verrons donc quels types d'obstacles cette nouvelle définition pourrait rencontrer et quels types de travaux seront nécessaires à une élaboration plus détaillée d'un tel concept.

\subsection{Idéalisation, abstraction et théorie idéale}

Le fait de concevoir l'autonomie personnelle en se fondant uniquement sur les compétences de la raison individuelle pose problème quant à l'interprétation des motivations à l'action. En effet, bien qu'on puisse imaginer que des individus puissent adhérer rationnellement à des principes de justice tels que ceux énoncés par Rawls, on voit mal ce qui les pousserait, dans le monde non idéal dans lequel nous sommes, à effectivement agir selon ces principes. On se retrouve ainsi face à un dilemme classique de la motivation à l'action. Bien que la raison puisse jouer un rôle motivationnel, elle n'est pas l'unique instigatrice des actes. Certains pourraient penser qu'une telle affirmation revient à rejeter la théorie idéale au profit d'une théorie non idéale qui tiendrait uniquement compte de données empiriques. Toutefois, affirmer que la raison n'est pas la seule source de motivation, ce n'est pas pour autant écarter la nécessité d'élaborer des théories idéales du genre de celle que Rawls propose. Notre proposition de modification du concept d'autonomie n'est pas un rejet des théories idéales en tant que tel, mais plutôt une prise de position par rapport à la façon dont ces théories doivent être construites pour ensuite instruire la théorie non idéale ou le design institutionnel. Dans la théorie idéale de Rawls, ce n'est pas seulement le concept de justice qui est idéalisé, mais aussi celui des agents qui, par leur rationalité, le fondent. Par conséquent, certains éléments jugés impertinents sont écartés de la conception idéale de l'agent. Or à quoi correspond une telle conception de la justice si elle ne provient pas d'une prise en considération de l'ensemble des caractéristiques humaines fondamentales, mais uniquement d'une caractéristique jugée suffisante, la raison? La justice est un concept qui devrait embrasser un pan plus large de l'expérience humaine.

La question plus large qui se dégage de ce constat est celle du rôle assigné à une telle théorie idéale. Selon John Simmons, dans la théorie de Rawls, 
«tandis que la théorie idéale dicte l'objectif, la théorie non idéale dicte la route vers cet objectif 35 ". Pour dicter un objectif, cependant, il faut se baser sur une certaine conception de la réalité, un certain diagnostic de la situation où l'on se trouve. Cet impératif relève en fait du problème général du fondement de la normativité. Le discours normatif, tout orienté qu'il soit vers l'avenir idéal, n'en demeure pas moins un discours émanant d'une réalité spécifique. Pour avoir un contenu qui légitime sa normativité, c'est-à-dire pour qu'il y ait un lien entre la situation présente et la norme visée, il doit s'ancrer dans une analyse au moins minimale de la situation de départ. L'emploi de concepts idéalisés tend à éviter cette étape diagnostique.

Si toute une tradition philosophique, à la suite de Kant, a tenté d'élaborer ses théories par le constructivisme moral, c'est d'abord pour s'assurer que ses théories soient les plus impartiales possibles, ensuite, pour qu'elles s'inscrivent dans la raison que chaque individu a à la fois en lui et en commun avec le reste de l'humanité et, enfin, pour éviter tout type de relativisme quant aux notions morales ou politiques fondamentales (telle que la justice). Cependant, cette façon de construire une base conceptuelle pose problème. Dans le processus d'élaboration des concepts, comme l'a si bien vu Onora O’Neill, il y a deux procédés possibles: l'idéalisation et l'abstraction ${ }^{36}$. O'Neill emploie elle-même cette distinction lorsqu'elle discute du libéralisme politique de Rawls ${ }^{37}$. Selon son analyse, les conditions qu'impose Rawls à sa conception du citoyen et de la société ne sont pas simplement une abstraction mais bien une idéalisation. Si l'idéalisation est utile dans la construction théorique, il faut l'utiliser avec précaution, nous dit-elle, surtout dans le cas du raisonnement pratique (qui est celui de toute philosophie politique), puisque la normativité inhérente aux concepts philosophiques rend l'épreuve de validation des concepts beaucoup moins évidente ${ }^{38}$.

Toutefois, il existe une autre tradition de pensée, avec Hegel pour chef de file, qui est moins bien représentée aujourd'hui dans l'élaboration des théories politiques. Il y a au cœur du projet politique de Hegel une méthodologie qu'il vaut la peine de remettre au goût du jour, ne serait-ce que parce qu'elle nous permettrait de jeter un regard nouveau sur des problématiques complexes. C'est du moins ce que soutient Axel Honneth ${ }^{39}$. L'auteur emploie l'expression «reconstruction normative " pour désigner cette approche typiquement hégélienne. Il la décrit ainsi: «les rapports modernes de vie sont

35. «Ideal and Nonideal Theory», A. J. Simmons, Philosophy and Public Affairs 38, $\mathrm{n}^{\circ}$ I (2OIO), p. I 2.

36. Cf. «Abstraction, Idealization and Ideology in Ethics ", O. O’Neill, Royal Institute of Philosophy Lecture Series, 22 (1987).

37. «Political Liberalism and Public Reason: A Critical Notice of John Rawls, Political Liberalism ", O. O’Neill, The Philosophical Review I06, $\mathrm{n}^{\circ} 3$ (1997).

38. Ibid., p. 4I9.

39. Les pathologies de la liberté: Une réactualisation de la philosophie du droit de Hegel, A. Honneth, Paris, La Découverte, 2008. 
reconstruits [...] d'une manière normative qui est telle qu'elle permet de mettre au jour les cadres d'interaction qui peuvent valoir comme des conditions indispensables à la réalisation de la liberté individuelle de tous les membres de la société ${ }^{40}$ ». Il s'agit donc d'abord d'enquêter sur les interactions sociales pour dégager leur structure et ainsi être capable d'en repérer les failles. Cette méthode repose sur la dialectique hégélienne selon laquelle les erreurs, les anomalies, les incohérences et la souffrance historiquement vécue déterminent l'orientation normative de l'Histoire. Sans adopter le point de vue métaphysique de Hegel sur la marche de l'Histoire, il est possible de reconnaître que le diagnostic d'une souffrance vécue (que ce soit la souffrance de l'ouvrier chez Marx ou le déni de reconnaissance chez Honneth) peut mener à l'identification des solutions possibles à cette souffrance. Selon cette logique, la définition de la justice se trouvera dans l'appréhension de l'injustice telle qu'elle est vécue par les individus. Alors que le constructivisme d'inspiration kantienne se fonde sur une expérience de pensée pour établir des normes, l'approche hégélienne repose donc sur un diagnostic de la réalité. Non seulement le fait de se fonder sur une description la plus précise possible de la réalité rend-il plus adéquats les concepts que nous employons, mais il assure que la théorie idéale, aussi abstraite soit-elle, puisse éventuellement informer une théorie non idéale plus efficace. La notion d'autonomie relationnelle s'inspire d'une telle méthodologie en se modelant sur une conceptualisation la plus réaliste possible des déterminants de l'autonomie.

\subsection{Un concept d'autonomie relationnelle sans surdétermination: une impossibilité?}

J'ai montré en quoi l'argument de Rawls en faveur d'une théorie idéale ne justifie pas la minceur conceptuelle de son concept d'autonomie. On pourrait toutefois répondre que les raisons principales pour lesquelles Rawls ne donne pas plus de chair à ce concept (ou même à son concept plus général de personne, dans le cas de Libéralisme politique) visent, d'une part, à éviter de faire entrer la conception de la personne dans une doctrine compréhensive spécifique et, d'autre part, à ne pas dénaturer le concept d'autonomie qui a traditionnellement servi de contrepoids individualiste aux tendances majoritaires de certains principes plus axés sur la communauté. Ce sont deux sérieux contre-arguments. Il est pourtant possible d'y répondre de façon satisfaisante.

\subsubsection{Le problème de la doctrine compréhensive}

Un des arguments contre l'emploi d'un concept d'autonomie plus empirique, tel que le proposent les approches relationnelles est que l'autonomie serait un concept qu'il ne faut pas surcharger de signification. Selon cet argu-

40. Ibid., p. 97. 
ment, si l'on définit le concept de manière trop spécifique (par l'ontologie relationnelle, entre autres), il sera nécessairement lié à une doctrine compréhensive. La fausseté de cet argument est double. D'abord, il est naif de croire que la définition de l'autonomie comme capacité rationnelle de l'agent également partagée entre tous est une description moins déterminée ou chargée qu'une autre. Elle est certainement conceptuellement moins épaisse, moins riche. Il n'empêche qu'elle ne représente pas moins une conception spécifique de l'individu, égale en ce point à une conception fondée sur une description psychologique et relationnelle. Dans les deux cas, une décision est certainement prise par ceux qui endossent l'une ou l'autre des conceptions, bien que toutes deux se veuillent neutres. Ce qui nous mène à la deuxième fausseté de l'argument: le fait d'avoir peur de glisser dans une doctrine compréhensive relève d'une distinction entre valeurs privées et valeurs publiques qui ne tient pas la route. Le rôle de la doctrine non compréhensive dans la théorie rawlsienne est de laisser un espace au consensus hors des croyances ou théories spécifiques auxquelles les individus adhèrent. Or, comme l'a pointé la critique communautarienne, on peut difficilement imaginer que les individus puissent faire abstraction de leurs valeurs privées, tirées de la doctrine compréhensive à laquelle ils adhèrent, lors des débats publics ou politiques. Il apparaît improbable que l'on puisse distinguer, au sein d'une même personne, des valeurs relevant du domaine privé et des valeurs politiques distinctes. Par conséquent, il devient difficile de déterminer ce qui découle d'une doctrine, compréhensive ou non. Si la distinction entre les deux types de doctrine a une valeur heuristique, il n'en demeure pas moins que la ligne qui les distingue est très floue. Il s'agit d'une raison suffisante pour ne pas tenir compte de cette catégorisation.

John Christman est probablement l'auteur qui a défendu de façon la plus explicite un concept individualiste et procédural d'autonomie contre la conception relationnelle ${ }^{41}$. Son argument consiste à dire que, tout en acceptant la thèse du moi socialement construit, il est possible et même nécessaire de s'en tenir à un concept traditionnel d'autonomie pour préserver son rôle théorique et normatif. En rester à un concept individualiste d'autonomie permettrait selon Christman d'éviter, entre autres, le perfectionnisme d'une idéalisation de la réalisation de soi-même et du gouvernement de soi. Sa critique du perfectionnisme s'appuie plus spécifiquement sur la conception de l'autonomie relationnelle que défend Marina Oshana ${ }^{42}$. Oshana s'oppose aux définitions purement internalistes de l'autonomie, notamment en raison

41. Cf, entre autres: «Relational Autonomy, Liberal Individualism and the Social Constitution of Selves", de J. Christman, Philosophical Studies II 7 (2004). Voir aussi «Autonomie individuelle et moi social ", Christman.

42. Christman fait référence à M. Oshana, «Personal Autonomy and Society », Journal of Social Philosophy 29, $\mathrm{n}^{\circ}$ I (I998). Pour une exposition plus détaillée et récente des arguments de Oshana, cf. Personal Autonomy in Society, M. Oshana, Aldershot, Engl., Ashgate, 2006. 
du fait qu'elles permettent de considérer la servitude volontaire comme une forme d'autonomie. Elle propose donc une définition socio-relationnelle qui aurait comme condition, en plus des prérequis psychologiques, certaines clauses de nature externe, comme la possibilité d'avoir accès à plusieurs options et certaines caractéristiques socio-relationnelles assurant que l'individu ne soit pas vulnérable et sous l'influence d'un autoritarisme indu. Pour Christman, une telle conception de l'autonomie est perfectionniste, puisqu'elle nécessite des relations sociales égalitaires pour être mise en place. En effet, Oshana pose comme condition que les individus soient dans une situation où ils puissent poursuivre des objectifs différents de ceux qui sont en autorité audessus d'elles. Selon Christman, cette clause mène directement à la promotion de relations sociales égalitaires comme conditions de l'autonomie et, par conséquent, elle fait de l'autonomie un concept intrinsèquement perfectionniste en ce sens qu'il contient en lui-même les germes d'un projet politique compréhensif.

Plusieurs problèmes se posent avec l'accusation de perfectionnisme que Christman fait à Oshana et qu'il étend à toutes les conceptions relationnelles de l'autonomie. La description que fait Christman du perfectionnisme est la suivante: il s'agit d'une «perspective qui implique que certaines valeurs intrinsèques - peut-être fondées dans la nature humaine - devraient guider l'action individuelle et sociale, indépendamment de l'endossement de ces valeurs par des individus minimalement rationnels et autonomes ${ }^{43}$ ». Il y a au moins deux éléments problématiques dans cette définition si l'on souhaite l'utiliser comme arme contre l'autonomie relationnelle et en défense d'un concept individualiste. Le premier est celui de la définition des «valeurs intrinsèques » qui sont "peut-être fondées dans la nature humaine ». On comprend mal en quoi l'autonomie relationnelle est différente sur ce point du déontologisme classique qui règne au sein des théories libérales. En effet, si les droits humains, par exemple, ne sont pas des valeurs intrinsèques fondées dans la nature humaine, on voit mal ce qu'ils sont. La défense même de l'autonomie, de la démocratie, de la dignité humaine, dépend fondamentalement d'une interprétation de la nature humaine. C'est parce que l'être humain est rationnel et autonome (aussi mince et procédural que soit le concept) que ces valeurs sont promues. Et elles le sont, dans toute doctrine libérale, même celle qui se veut la plus antiperfectionniste possible. Même les approches conséquentialistes doivent se fonder sur des caractéristiques humaines fondamentales comme valeurs intrinsèques pour pouvoir opérer (l'évitement de la souffrance, le bien-être, etc.). Le deuxième élément problématique est celui qui pourfend l'utilisation de telles valeurs intrinsèques en

43. "Relational Autonomy, Liberal Individualism and the Social Constitution of Selves", Christman, p. I 52. "[S] uch a view implies that there are certain intrinsic values grounded in human nature perhaps - that should guide individual and social action independent of the endorsement of those values by minimally rational, autonomous individual. » 
vue d'élaborer un discours normatif. Bien sûr, une conception relationnelle de l'individu ne souhaite pas imposer aux individus des façons de faire ou de penser, puisqu'elle souhaite préserver le concept d'autonomie. Mais, de toute évidence, des conclusions morales, et donc normatives, peuvent être tirées de la façon dont on conçoit et décrit la constitution de l'autonomie. C'est précisément le génie de l'architecture de la philosophie pratique de Kant, qui tire sa morale de sa conception de l'autonomie, de procéder ainsi.

En somme, la conception relationnelle de l'autonomie n'est pas plus compréhensive ou perfectionniste que la conception rationaliste. Toutes deux sont des descriptions des agents, basées sur certaines expériences ou faits. La différence principale entre les deux est que, puisque la conception relationnelle est plus étoffée et plus proche de la façon dont se vit vraiment l'autonomie, elle permet de mieux expliquer certains phénomènes, dont la coopération sociale, et par conséquent mènera probablement à des conclusions normatives différentes. Mais, au final, elle ne demeure qu'un concept descriptif dont l'élaboration a pour but de donner les meilleurs outils conceptuels possibles à qui se voudra l'architecte d'une théorie de la justice qui rende compte de toutes les dimensions possibles des vulnérabilités individuelles. Car une des forces du concept relationnel d'autonomie est de théoriser les conditions de l'autonomie et, par conséquent, de mieux expliciter les dangers auxquels elle est exposée ${ }^{44}$. Bien sûr, pour ce faire, il décrit de façon spécifique les relations interpersonnelles et sociales. En ce sens, il est soumis aux mêmes périls que toute théorie politique ou même que toute philosophie. Et comme pour ses semblables, la clé pour éviter la réification est le travail théorique constant, en rapport dialogique avec la réalité pratique, afin de constamment ajuster ses présupposés aux modifications du réel et du savoir. En ce sens, le concept relationnel d'autonomie est appelé à évoluer avec les sciences sociales et peutêtre plus spécifiquement avec la psychologie. Mais, dans la mesure où il représente une conceptualisation de la réalité dans l'objectif de construire une théorie normative, il reste éloigné de toute conception du bien en tant que telle.

Même Rawls, dans son libéralisme politique, n'échappe pas lui-même à l'ambiguïté engendrée par le concept d'autonomie quant au danger du perfectionnisme. En effet, sa description de l'autonomie politique par l'adéquation de la rationalité et du sens de la justice provient tout aussi bien d'une conception spécifique de l'individu. La notion d'autonomie est intrinsèquement ambiguë, et c'est précisément ce qui fonde la problématique à laquelle nous nous intéressons. D'une part, on peut concevoir l'autonomie d'une

44. C’est à partir de cette caractéristique spécifique aux approches de l'autonomie relationnelle que Garrau et Le Goff élaborent leur critique du néorépublicanisme, dont elles jugent le formalisme inapte à rendre compte de ces vulnérabilités. Cf. "Vulnérabilité, non-domination et autonomie: vers une critique du néorépublicanisme", M. Garrau et A. Le Goff, Astérion, L'ami et l'ennemi, $\mathrm{n}^{\circ} 6$ (2009). 
manière plus minimale, qui consisterait à affirmer que tous les êtres humains sont autonomes parce que tous sont rationnels et tous effectuent certains choix. Cette définition peut être purement opérationnelle, dans la mesure où ceux qui l'utilisent sont conscients que la réalité n'est pas aussi simple, mais qu'à des fins de théorisation et dans le contexte politique l'autonomie doit être comprise comme telle. Toutefois, employer le terme autonomie en ce sens, même de façon simplement opérationnelle, est une dénaturation du concept due à une influence kantienne voulant que la rationalité se confonde avec la liberté. Si tel est vraiment le concept d'autonomie, il ne se distingue pas substantiellement du concept de liberté ou d'indépendance. D'autre part, comme c'est le cas dans notre argument, on peut supposer que l'autonomie est un phénomène bien spécifique d'autodétermination qui nécessite certaines conditions émotionnelles et sociales, et qui n'est jamais totalement et finalement accompli. Dans ce cas, il nous faut prendre acte des spécificités de ce terme, ce qui nous permet de le différencier clairement des concepts de liberté et d'indépendance ${ }^{45}$. Le concept a alors une richesse nouvelle. Employé comme prémisse d'une théorie de la justice, il pourrait ouvrir une toute nouvelle perspective sur les tenants et aboutissants d'une société juste.

\subsubsection{Une dénaturation du concept d'autonomie?}

Passons donc au deuxième argument contre l'autonomie relationnelle, soit celui de la dénaturation du concept. Il y a en fait deux versions de cet argument. La première est celle qui soutient que l'autonomie relationnelle est un paradoxe et que, ultimement, adopter un tel concept revient à nier l'autonomie. La deuxième consiste à reconnaître la pertinence de l'analyse psychologique individuelle et sociale que font les tenants de l'autonomie relationnelle, tout en refusant de l'employer en philosophie politique, afin de préserver la nature individualiste du concept et le contrepoids qu'il représente face aux intérêts de groupe. La première version peut trouver une réponse dans la description des fondements de l'autonomie relationnelle telle qu'elle est faite dans la première section de ce texte. En effet, bien que les tenants d'une telle conception soutiennent que l'autonomie est constituée en grande partie par les relations sociales dans lesquelles elle s'inscrit, il est possible de décrire l'autonomie relationnelle de façon plus nuancée. Si l'on se fie à la fois aux tra-

45. Par ailleurs, je crois que définir ainsi l'autonomie permet de mieux comprendre le dilemme entre la liberté négative et la liberté positive telles que définies par Berlin. En effet, un tel concept d'autonomie inclut en lui-même la liberté positive et, ce faisant, nous donne les moyens de mieux la définir. C'est aussi ce que pense Emmanuel Renault: «L'un des enjeux les plus immédiats de l'idée d'autonomie est bien de savoir si le social peut jouer positivement (et non pas seulement par défaut) un rôle d'obstacle non pas simplement à la liberté négative, mais également à ce type de liberté positive, rationnelle et réflexive, qui semble constituer la forme de liberté la plus haute " ("Modèles du social et modèles de l'autonomie », E. Renault, in Comment penser l'autonomie? Entre compétences et dépendances, sous la dir. de M. Jouan et S. Laugier, Paris, Presses universitaires de France, 2009, p. 259). 
vaux en psychologie, mais aussi à la théorie de la reconnaissance de Honneth, ce n'est pas de façon directe que le contexte relationnel conditionne l'autonomie, mais bien parce que le rapport aux autres influence le rapport à soimême. Or c'est bien dans cette relation à soi-même que, pour certains, s'exerce la véritable autonomie.

Répondre au deuxième argument concernant la dénaturation du concept d'autonomie est un peu plus complexe, puisqu'il s'agit ici d'évaluer le rôle que joue ce concept dans les théories de la justice et, de façon plus générale, le rôle historique qu'a occupé le concept d'autonomie en philosophie politique. Rappelons que, selon cet argument, bien que le moi soit effectivement constitué socialement, il n'est pas souhaitable d'employer le concept d'autonomie relationnelle dans le contexte de la théorie politique. Cet argument accepte la description relationnelle de l'autonomie et la façon dont celle-ci décrit la construction sociale du moi et parfois même du je. Il refuse cependant de faire entrer ces considérations dans l'élaboration et l'utilisation du concept d'autonomie tel qu'on l'emploie en philosophie politique sous prétexte que, d'une part, ce n'est pas nécessaire, et que, d'autre part, le rôle de protection de l'individu joué par le concept serait annulé. Nous reviendrons sur la question de la nécessité du concept d'autonomie relationnelle sous peu. Attardons-nous d'abord à l'argument qui fait appel au rôle du concept d'autonomie en philosophie politique.

L'apparition du concept d'autonomie a été l'aboutissement d'un processus par lequel, durant le siècle des Lumières, les philosophes moraux ont tenté de limiter la place donnée à l'influence de Dieu sur la vie terrestre ${ }^{46}$. Aujourd'hui toutefois, après l'expérience traumatique des totalitarismes du vingtième siècle, le rôle de l'autonomie dans les théories politiques tient plus du bouclier face à la menace constante des dérives majoritaires. D'une façon plus positive, on peut considérer qu'elle est surtout la condition fondamentale de la démocratie. En effet, la défense de la démocratie ne peut se faire que dans la mesure où une conception de l'autonomie individuelle la soustend. La démocratie est la forme politique qui reconnaît à chaque individu la capacité de raisonner, de prendre des décisions, de faire des choix et de fixer ses propres lois. C'est une entreprise politique à la fois de célébration de la capacité de l'être humain à l'autonomie, et de la réconciliation constante des autonomies personnelles et du pluralisme né de la diversité des façons dont elles s'incarnent. Dans ce contexte, et étant donné le consensus intellectuel sur l'importance de promouvoir des régimes politiques démocratiques,

46. Même si une des interprétations les plus communes de l'évolution de la morale au dix-huitième siècle est celle d'une sécularisation, S. B. Schneewind affirme qu'on ne peut pas sérieusement la soutenir. Les penseurs des Lumières auraient plutôt tenté de limiter la place de Dieu dans leurs explications, tout en le rendant indispensable à la moralité, soit en en étant la source, soit en servant de motivation à l'action morale. Cf. The Invention of Autonomy: A History of Modern Moral Philosophy, J. B. Schneewind, Cambridge, U. K., Cambridge University Press, I998, chapitre I. 
la complexification du concept d'autonomie proposé par l'approche relationnelle inquiète. Est-ce qu'une conception relationnelle de l'autonomie peut assumer tous ces rôles ? Répondre à cette question nous oblige à préciser la nature de notre compréhension de l'autonomie relationnelle et des théories qui en font la promotion.

Pour ce faire, revenons à la critique de John Christman. Un des arguments de Christman consiste à dire que les approches soutenant l'autonomie relationnelle ont tendance à réifier l'identité des individus et à ignorer la fluidité des parcours de vie. Cet argument relève de la lecture spécifique que fait Christman des perspectives relationnelles et plus précisément de la théorie de la reconnaissance de Honneth. Comme plusieurs, Christman considère le concept de reconnaissance comme un concept d'identification et, par conséquent, d'étiquetage. "Dire que la reconnaissance entre personnes est nécessaire à l'autonomie, c'est dire que, pour être autonome, il faut être reconnu — et, plus précisément, reconnu en tant que tel ou tel être social ${ }^{47}$.»Par conséquent, la reconnaissance fige toujours l'identité et peut, selon Christman, cristalliser l'appartenance sociale à certains groupes, évacuant ainsi la possibilité d'une réelle autonomie par rapport aux groupes d'appartenance. Plusieurs problèmes se posent dans la compréhension et la réfutation de cet argument. Le plus important est certainement la polysémie du terme reconnaissance et la différence des usages qui en sont faits par différents penseurs politiques. La reconnaissance ne signifie pas la même chose pour Axel Honneth, pour Charles Taylor ou pour Nancy Fraser, par exemple ${ }^{48}$. Dans le cas de Honneth, le terme reconnaissance a un sens plus classiquement hégélien et intrinsèquement moral. Il ne fait donc pas nécessairement référence à l'appartenance sociale, bien au contraire. Il explique les rapports moraux entre les individus de façon formelle et les liens dialectiques entre l'individu et sa communauté. En ce sens, on ne peut pas dire qu'un tel type de reconnaissance surdétermine l'identité de l'individu: il décrirait plutôt la façon dont l'identité personnelle se forme et change selon les circonstances sociales. La fluidité des caractéristiques personnelles est donc conceptuellement respectée. On voit par conséquent que le concept de reconnaissance de Honneth se distingue assez clairement de celui employé par les théoriciens multiculturalistes ou les tenants des politiques de la différence. Bien sûr, son approche n'est pas incompatible avec certaines thèses et revendications provenant de ces derniers. Toutefois, je crois que la force d'une telle théorie est précisément d'éviter de devoir réifier les identités spécifiques ou de définir les

47. «Autonomie individuelle et moi social», Christman, p. I94.

48. Le débat entre Honneth et Fraser le prouve bien. Cf. Redistribution or recognition? A political-philosophical exchange, N. Fraser et A. Honneth, London, Verso, 2003. Simon Thompson a pour sa part bien montré les différences fondamentales entre les approches de Honneth, Fraser et Taylor dans The Political Theory of Recognition: A Critical Introduction, S. Thompson, Cambridge, UK; Malden, MA, Polity, 2006. 
individus uniquement dans leur rapport à leurs groupes d'appartenance. En se penchant plus spécifiquement sur la façon dont le rapport psychologique à l'autre est vécu et sur l'effet de cette expérience sur les motivations et les actions des individus, on peut soutenir une conception relationnelle de l'autonomie selon laquelle l'autonomie authentique ne peut s'exercer que dans certains paramètres spécifiques de reconnaissance, sans toutefois figer les individus dans une identité essentialisée.

Christman avance un dernier argument contre l'autonomie relationnelle, celui-ci plus spécifiquement contre l'utilisation de cette notion comme concept politique. Dans ses mots,

dire qu'[une personne dans une situation d'obéissance stricte à une autorité] n'est pas autonome implique qu'elle ne jouit pas du marqueur du statut de citoyen indépendant, dont la perspective et l'orientation des valeurs trouvent écho dans les processus démocratiques qui constituent une politique sociale légitime ${ }^{49}$.

Si l'on s'inspire de la définition d'Oshana, une personne sous autorité qui n'a pas les moyens de remettre en cause cette autorité si elle le désire n'est pas autonome. Or affirmer une telle définition revient à dire que les individus les plus vulnérables ne sont pas autonomes. Si l'on accepte que c'est grâce à la reconnaissance de l'autonomie des individus que chacun se voit conférer des droits civiques et politiques, alors adopter la notion d'autonomie relationnelle, selon cette perspective, voudrait dire refuser aux personnes les plus vulnérables leur droit de participation égale à la société sous prétexte qu'elles ne sont pas autonomes.

Le problème, c'est que cet argument qui, à la première lecture, peut sembler très raisonnable, est bourré de présupposés. Le plus important est celui selon lequel l'autonomie est l'élément à partir duquel on juge une personne apte à participer à la vie commune ou à obtenir des droits civiques et politiques. Bien sûr, historiquement, c'est parce que l'on a reconnu la capacité des individus à l'autonomie que l'on a universalisé les droits civiques et politiques. Mais il serait aberrant de penser qu'on suppose pour cela qu'il y a une adéquation parfaite entre l'autonomie d'une personne et son droit de participation. De nombreuses personnes ne sont pas autonomes, même au sens réduit et procédural que défend Christman, et conservent malgré tout légitimement leurs droits civiques. Il y a certainement d'autres raisons possibles que son identification comme personne autonome pour assigner des droits à un individu. Pour explorer ces voies, il faudrait toutefois sortir du paradigme kantien selon lequel la dignité humaine repose sur la possession

49. "Relational Autonomy, Liberal Individualism and the Social Constitution of Selves ", p. I 57, Christman. "But to say that she is not autonomous implies that she does not enjoy the status marker of an independent citizen whose perspective and value orientation get a hearing in the democratic processes that constitute legitimate social policy. » 
effective d'une autonomie morale et, par conséquent, sur la rationalité des individus. Une telle conception a certes été historiquement importante, mais elle mérite toutefois d'être revisitée à l'aune des avancées contemporaines en sciences humaines et sociales. Une vision plus complexe et plus riche de l'être humain, qui reconnaît à la fois sa rationalité et son émotivité, sa particularité d'individu et son caractère fondamentalement social, doit-elle nécessairement abandonner le concept de dignité humaine et par conséquent l'affirmation politique et juridique de cette dignité ? Il est évident que non. Il n'est pas absolument nécessaire de faire dépendre de la pleine autonomie rationnelle des individus le droit de participer à la vie démocratique. Entre autres parce que la participation à la vie collective est un élément essentiel de l'autonomie individuelle. C'est d'ailleurs précisément ce que suggère une conception relationnelle et formelle de l'autonomie. La démocratie est une forme de rapport de reconnaissance mutuelle qui respecte l'autonomie individuelle en ce sens qu'elle permet l'autonomie des individus qui y participent.

Par ailleurs, l'objectif du concept d'autonomie relationnelle n'est pas d'être capable d'identifier les personnes autonomes et celles qui ne le sont pas, ou de leur vouer un degré de respect différent. Malgré toutes les définitions possibles de l'autonomie, il demeure toujours approximatif de mesurer un «niveau » d'autonomie. À défaut de pouvoir déterminer le niveau d'autonomie de chacun, les théories politiques doivent considérer les individus comme égaux dans leur capacité à l'autonomie, et que cette égalité suffit à la défense d'un régime démocratique et de l'égalité des droits. D'autant plus que cette égalité des droits est une condition de l'autonomie relationnelle. La différence entre un concept individualiste et un concept relationnel d'autonomie dans la pensée politique ne relève donc pas du rapport des individus à la démocratie, mais plutôt de l'élaboration des principes de justice. Notre hypothèse est donc plutôt qu'en ayant un meilleur outil conceptuel entre les mains, un concept d'autonomie qui soit plus adéquat pour définir la relation à soi-même, la façon dont on pense l'agentivité humaine se modifiera. Par conséquent, l'ensemble des descriptions concernant les interactions sociales se trouvera teinté d'une nouvelle couleur.

\subsection{Fonder la coopération sociale dans le rapport à soi-même}

Une fois que l'on a accepté la pertinence de penser que l'autonomie se construit de façon relationnelle et que l'on peut employer un tel concept dans l'élaboration de théories de la justice sans risquer de défendre une théorie perfectionniste, il reste à montrer pourquoi il est nécessaire de l'adopter. Une partie de la réponse est qu'un concept relationnel de l'autonomie permet une meilleure compréhension de la motivation à la coopération sociale. Parce qu'elle est fondée sur le fait que les rapports aux autres influencent, modifient et constituent en partie le rapport à soi-même, l'approche relationnelle reste fidèle à l'objectif d'un idéal d'autonomie, soit une émancipation de 
tout ce qui peut étouffer ou brouiller le rapport à soi-même qui permet aux individus de délibérer. Les féministes ou les penseurs de la théorie critique cherchent tous à promouvoir la possibilité pour chacun d'effectuer des choix, de se respecter soi-même, de se réaliser. En ce sens, ils ne se distinguent pas du libéralisme. Ce qui les motive à critiquer le libéralisme, c'est plutôt la prise de conscience que ce rapport à soi ne dépend pas uniquement de qualités intérieures, mais se construit dans le rapport au monde, et cette caractéristique doit être représentée dans les outils analytiques que l'on emploie pour élaborer des normes. Si l'autonomie, aussi bien pour les tenants d'une approche procédurale que pour les tenants d'une approche relationnelle, est une capacité à réfléchir sur soi-même, elle ne peut se faire indépendamment du regard de l'autre. Christman reconnaît lui-même que l'autonomie en tant qu'autoréflexion consiste en «l'habileté d'évaluer les différents aspects de son être et la libération des facteurs et conditions indépendants dont nous savons qu'ils empêchent effectivement la compréhension minimale de soi ${ }^{50}$ ». Il reconnaît donc la double composition de l'autonomie, à la fois déterminée par des critères internes et des critères externes. L'originalité de l'autonomie relationnelle est de les lier en faisant dépendre les critères internes (ou du moins, certains d'entre eux) des critères externes.

Ce faisant, l'ontologie relationnelle permet de fonder dans le rapport à soi la motivation à la coopération sociale. En effet, la prise de conscience de l'interdépendance forte qui nous lie donne une raison beaucoup plus tangible aux individus de collaborer. Pour les individus, la conscience que l'exercice de leur autonomie leur est accordé, en plus de la reconnaissance juridique de leurs droits, nécessite certaines conditions de base. Lorsqu'ils s'aperçoivent que leur propre capacité d'effectuer des choix, de faire des plans de vie, de se réaliser dépend de leurs relations intimes autant que de leurs relations sociales, il semble plus probable qu'ils en viennent à adopter des comportements coopératifs. Il ne s'agit alors ni d'altruisme ni d'égoïsme, puisque la conscience qu'à grande échelle les intérêts de tous sont liés brouille cette distinction. Il reste encore beaucoup de travail à faire pour mieux détailler en quoi une telle notion d'autonomie relationnelle permettrait de mieux saisir la dynamique des rapports sociaux. Toutefois, nous espérons avoir montré de façon convaincante qu'il y a une faille dans notre façon actuelle de penser l'agentivité individuelle et qu'il serait avantageux sur le plan théorique d'y remédier afin de viser une philosophie politique plus proche des préoccupations sociales réelles.

50. Ibid., p. I 54. «This [l'autoréflexion nécessaire à l'autonomie] includes ability to assess the various aspects of one's being, and the freedom from those factors and conditions that we know independently effectively prevent minmal self-understanding. » 


\section{Conclusion}

L'introduction de la notion d'autonomie relationnelle dans les prémisses d'une théorie de la justice est un projet à long terme puisqu'il nécessiterait d'abord une conceptualisation adéquate du terme. Cependant, il s'agit d'une entreprise non seulement possible, mais nécessaire. Notre compréhension de la subjectivité des individus a beaucoup évolué depuis quelques décennies, et il est important que ces changements se reflètent dans les théories politiques. Dans la défense de l'autodétermination individuelle au regard de la perspective relationnelle, il y a aussi un rappel des limitations du pouvoir motivationnel de la rationalité. Il ne s'agit pas de nier que la raison puisse être à l'origine de certains actes, mais de voir que, d'une part, elle n'agit souvent pas seule en tant que motivation et, d'autre part, qu'elle-même n'est jamais totalement pure et indépendante de l'expérience émotionnelle engendrée par les relations interpersonnelles et sociales. Étant donné que la philosophie politique contemporaine cherche à définir les paramètres du vivre-ensemble et de la collaboration entre les individus, il est important qu'elle se dote d'une compréhension adéquate de la psychologie morale des agents et, par conséquent, qu'elle tienne compte des dimensions non rationnelles de l'individu. Cette ambition peut bien sûr se révéler complexe, puisque la philosophie elle-même fonctionne à partir du raisonnement rationnel. Mais il faut éviter de mélanger la façon dont la réflexion philosophique est menée et la façon dont elle décrit son objet. En tant qu'objet de réflexion, l'être humain ne peut pas être dépouillé de certaines autres caractéristiques fondamentales sous prétexte que ces traits ne correspondent pas au discours rationnel auquel la philosophie aspire. Il y a certainement lieu de discuter rationnellement et objectivement de la dimension affective des rapports humains.

Utiliser une approche relationnelle pour décrire l'autonomie est une façon de tenter de rendre compte de la complexité des rapports humains dans le cadre de nos réflexions politiques. Dans le cadre du paradigme démocratique, étant donné l'importance de la notion d'autonomie pour l'ensemble de la pensée politique contemporaine et pour la justification de nos institutions, il est essentiel de reconnaître l'étendue et la portée de l'interdépendance entre les individus, et entre les individus et les institutions. Il ne peut certes y avoir de démocratie sans la reconnaissance de la capacité des individus à l'autonomie. Mais, plus encore, il ne peut y avoir de véritable démocratie sans la conviction partagée d'une dépendance mutuelle incontestable. D'abord parce que la seule participation aux institutions démocratiques nécessite l'adhésion de chacun sinon à des normes communes, du moins à des procédures jugées équitables. Selon la description de l'agentivité ébauchée plus haut, la compréhension de cette équité ne peut pas se faire uniquement par une compréhension rationnelle des intérêts individuels, mais doit plutôt passer par un besoin de reconnaissance sociale, par une prise de conscience d'une forme d'interdépendance fondamentale et par un désir d'exercer son autonomie à travers la participation à une entreprise collective. Et il 
faut voir que, pour les tenants d'une conception relationnelle de l'autonomie, la possibilité de participer aux délibérations collectives n'est pas qu'une forme de l'expression de l'autonomie, c'est une de ses conditions fondamentales.

C'est ultimement l'argument le plus puissant en faveur d'une conception relationnelle de l'autonomie: reconnaître que l'autonomie est plus qu'une capacité rationnelle, mais représente aussi une situation sociale et affective spécifique permet de comprendre les rapports complexes qu'entretiennent l'individu et la société. Elle permet aussi de pointer avec plus de précision les manques, les vulnérabilités dont certains individus sont victimes et d'expliquer leur souffrance à partir de leur capacité à être autonome. L'utilisation d'une notion relationnelle d'autonomie nous amène alors à considérer l'exclusion sociale dans une toute nouvelle perspective. Il ne s'agit plus uniquement de dire que l'injustice provient du fait que l'individu est dépourvu de certains droits spécifiques ou, dans le cas de la pauvreté par exemple, qu'il souffre d'un problème de redistribution des richesses, mais d'élargir la définition de la justice à la souffrance sociale et, ultimement, à la souffrance psychique $^{51}$. Car en considérant l'effet de la reconnaissance sur le rapport à soi-même, on en vient à comprendre jusqu'à quel point les rapports sociaux sont déterminants dans l'expérience de l'injustice. Les obligations qui naissent d'une telle description de l'autonomie individuelle sont donc beaucoup plus fortes que celles qui proviennent d'une description rationnelle de l'équité. Il ne s'agit pas seulement de donner aux individus les moyens matériels pour qu'ils puissent se développer comme ils l'entendent, mais bien plutôt de les reconnaître comme membres à part entière d'une communauté pour leur permettre d'être pleinement autonomes. Par ailleurs, ultimement, le problème de la redistribution des richesses dépend de situations de reconnaissance. S'il y a des laissés-pour-compte dans le système économique, c'est parce qu'ils ne sont généralement pas considérés et valorisés par la société et le système actuel de redistribution des richesses. Et le fait que l'on tolère encore aujourd'hui les souffrances inacceptables de populations entières pour assurer la stabilité de l'économie mondiale relève nécessairement d'un déni de reconnaissance des gens qu'on ne considère pas comme nos semblables.

\section{Bibliographie}

Ralph Adolphs. "Cognitive Neuroscience of Human Social Behaviour", Nature Review Neuroscience 4, 2003, I65-178.

Joel Anderson et Axel Honneth. "Autonomy, Vulnerability, Recognition, and Justice ", in Autonomy and the Challenges to Liberalism: New Essays, sous la dir. de J. Christman et J. Anderson, I27-I49, Cambridge, UK; New York, Cambridge University Press, 2005.

John Bowlby. L'attachement, Paris, Presses Universitaires de France, I969.

51. Cf. L'expérience de l'injustice: reconnaissance et clinique de l'injustice, E. Renault, Armillaire, Paris, Découverte, 2004. 
Inge Bretherton. "Attachment Theory: Retrospect and Prospect ", Monographs of the Society for Research in Child Development 50, $\mathrm{n}^{\circ}$ I, I985, 3-35.

Jerome S. Bruner. Actual Minds, Possible Worlds, Cambridge, Mass., Harvard University Press, I986.

John Christman. "Autonomie individuelle et moi social", in Comment penser l'autonomie: entre compétences et dépendances, sous la dir. de M. Jouan et S. Laugier, I69-20I, Paris, Presses universitaires de France, 2009.

. "Relational Autonomy, Liberal Individualism and the Social Constitution of Selves ", Philosophical Studies I I7, 2004, I43-I64.

John Christman et Joel Anderson (dir.). Autonomy and the Challenges to Liberalism: New Essays, Cambridge, UK, Cambridge University Press, 2005.

Antonio Rosa Damasio. L'erreur de Descartes, Paris, Éditions O. Jacob, I994.

R. J. Dolan. "Emotion, Cognition and Behaviour", Science 298, n 5596, 2002, II9I-II94.

Nancy Fraser et Axel Honneth, Redistribution or recognition? A political-philosophical exchange, London, Verso, 2003.

Marie Garrau et Alice Le Goff. "Vulnérabilité, non-domination et autonomie: vers une critique du néorépublicanisme", Astérion, L'ami et l'ennemi, n $\mathrm{n}^{\circ}$ 6, 2009.

Bénédicte Giffard et Bernard Lechevalier. "Neurosciences et affects ", Champ Psychosomatique 4I, $\mathrm{n}^{\circ}$ I, 2006, I I-27.

Alison Gopnik. The Philosophical Baby: What Children's Minds Tell Us About Truth, Love, and the Meaning of Life, Ist, New York, Farrar, Straus and Giroux, 2009.

Axel Honneth. Les pathologies de la liberté: Une réactualisation de la philosophie $d u$ droit de Hegel, Paris, La Découverte, 2008.

. The Struggle for Recognition: The Moral Grammar of Social Conflicts, trad. de J. Anderson, Cambridge, Mass., Polity Press, I995.

Robert Leckey. Contextual Subjects: Family, State and Relational Theory. University of Toronto Press, 2008.

Catriona Mackenzie et Natalie Stoljar (dir.). Relational Autonomy: Feminist Perspectives on Autonomy, Agency, and the Social Self, Oxford University Press, USA, 2000.

Ian R. Macneil. «Relational Contract Theory: Challenges and Queries» Nw. UL Rev. 94, I999, 877.

Peter Marris. The Politics of Uncertainty: Attachment in Private and Public Life, New York, Routledge, I996.

Onora O’Neill. "Abstraction, Idealization and Ideology in Ethics ", Royal Institute

of Philosophy Lecture Series 22, I987, 55-69.
. "Political Liberalism and Public Reason: A Critical Notice of John Rawls, Political Liberalism ", The Philosophical Review I06, n 3, I997, 4I I-428.

Marina Oshana. "Personal Autonomy and Society ", Journal of Social Philosophy 29, $\mathrm{n}^{\circ}$ I (I998), 8 I-IO2.

- Personal Autonomy in Society, Aldershot, Engl., Ashgate, 2006.

Emmanuel Renault. L'expérience de l'injustice: reconnaissance et clinique de l'injus-

tice, Armillaire, Paris, Découverte, 2004.
. "Modèles du social et modèles de l'autonomie ", in Comment penser l'autonomie? Entre compétences et dépendances, sous la dir. de M. Jouan et S. Laugier, 253-268, Paris, Presses universitaires de France, 2009. 
Françoise Schenk. "Les émotions de la raison», Revue européenne des sciences sociales, tome XLVII, $\mathrm{n}^{\circ}$ I44, 2009, I 5 I-I 62.

J. B. Schneewind. The Invention of Autonomy: A History of Modern Moral Philosophy, Cambridge, U.K., Cambridge University Press, 1998.

Allan N. Schore. "Effects of a Secure Attachment Relationship on Right Brain Development, Affect, Regulation, and Infant Mental Health», Infant Mental Health Journal 22, n I-2, 200I, 7-66.

A. John Simmons. "Ideal and Nonideal Theory", Philosophy and Public Affairs 38 , $\mathrm{n}^{\circ}$ I, 2010, 5-36.

Daniel N. Stern. The Interpersonal World of the Infant: A View from Psychoanalysis and Developmental Psychology, New York, Basic Books, 1985.

Charles Taylor. Les sources du moi: la formation de l'identité moderne, trad. de C. Melançon, $2^{\mathrm{e}}$ éd., Montréal, Boréal, 2003.

Charles Taylor. "The Dialogical Self» dans Rethinking Knowledge: Reflections Across the Disciplines, R. E. Goodman et W. R. Fischer (dir.), Albany State University of New York, I995, 57-66.

Simon Thompson. The Political Theory of Recognition: A Critical Introduction, Cambridge, UK; Malden, MA, Polity, 2006.

Marinus H. van IJzendoorn, Jarissa Dijkstra et Adriana G. Bus. "Attachment, Intelligence, and Language: A Meta-analysis », Social Development 4, $\mathrm{n}^{\circ} 2$ (I995), II 5-I 28. 